\title{
Liver Disease in Pregnancy and Transplant
}

\author{
Saad Alghamdi ${ }^{1}$. Jaquelyn Fleckenstein ${ }^{1}$
}

Published online: 25 July 2019

(C) The Author(s) 2019

\begin{abstract}
Purpose of Review The purpose of this review is to discuss current and new knowledge regarding liver disease in pregnancy and pregnancy post-liver transplantation.

Recent Findings Severe liver disease associated with pregnancy is rare. Liver biopsy is rarely needed for diagnosis but is safe in selected cases. Intrahepatic cholestasis of pregnancy (ICP) with serum bile acids level $>40 \mu \mathrm{mol} / \mathrm{L}$ is associated with adverse fetal outcomes. Ursodeoxycholic acid should be initiated at diagnosis. Portal hypertension can worsen during pregnancy and screening endoscopy should be performed in the 2 nd trimester. Maternal hepatitis B antiviral therapy can be considered in the $3 \mathrm{rd}$ trimester if HVB DNA > 200,000 IU/ml. Tacrolimus is the optimal immunosuppressive therapy during pregnancy post-transplantation. Preconception renal function predicts pregnancy outcome. Overall, the outcome of pregnancy post-transplantation is good but there is an increased risk of preterm delivery, low birth weight, hypertension, and pre-eclampsia.

Summary Liver disease of pregnancy can be divided into diseases unique to pregnancy, exacerbated by pregnancy or coexisting with pregnancy. Overall, the outcome of pregnancy post-liver transplantation is good.
\end{abstract}

Keywords Intrahepatic cholestasis · HELLP $\cdot$ AFLP $\cdot$ Viral hepatitis and pregnancy, pregnancy post-transplantation

\section{Introduction}

Liver dysfunction during pregnancy represents a unique challenge to health care providers. Early recognition and appropriate management are warranted to avoid potentially serious consequences for both the mother and fetus. Other major considerations are the teratogenic effect of certain medications and the risk pregnancy may pose on any underlying liver condition. Differential diagnosis include diseases unique to pregnancy, pregnancy in the setting of underlying chronic liver diseases, and coincidental conditions to pregnancy such as viral hepatitis, and Budd-Chiari [1]. Gallstones are commonly seen during pregnancy; however, gallbladder and biliary complications are beyond the scope of this review.

Liver diseases unique to pregnancy are seen in approximately $3 \%$ of all pregnancies. However, severe liver injury is encountered in a small subset of patients [2]. The incidence

This article is part of the Topical Collection on Liver

Jaquelyn Fleckenstein

jflecke@wustl.edu

1 Department of Internal Medicine, Washington University School of Medicine, St. Louis, MO, USA of liver diseases unique to pregnancy in the USA is $0.77 \%$ [3]. Symptoms may vary according to the severity of liver injury from abdominal pain, nausea, and vomiting to signs of acute liver failure. Common physical examination findings in pregnancy are palmar erythema and spider angiomas and their presence may not indicate an underlying chronic liver disease. Normal changes seen in pregnancy should be considered in the interpretation of liver function test (LFT). Alkaline phosphatase can be elevated in the third trimester due to placental production. Alpha fetoprotein (AFP) is not routinely obtained in this group of patients; however, it is produced by the fetal liver and commonly elevated [4]. Any elevation in aminotransferases, bilirubin, or prothrombin/INR, however, is considered abnormal and requires evaluation.

Liver ultrasound remains the best initial diagnostic imaging modality. It is available, safe, and cost effective. MRI without gadolinium is the preferred alternative if the ultrasound cannot be obtained or non-diagnostic. Gadolinium crosses the placenta and the fetus excretes it into the amniotic fluid. Therefore, its use is not recommended. The CT is less preferable due to the risk of radiation, although it can be obtained if there is no alternative in a select group of patients [5]. The diagnosis can be determined in a vast majority of cases by clinical, laboratory, and imaging parameters. Liver biopsy is rarely needed to make the diagnosis. However, per 
American College of Gastroenterology guidelines, liver biopsy can be performed safely during pregnancy when required [6]. A recent study evaluated pregnancy outcome in 23 patients who underwent a liver biopsy during pregnancy. This study demonstrated no increased risk of bleeding or stillbirth. There was a moderate increased risk of preterm birth and small for gestational age [7].

\section{Liver Diseases Unique to Pregnancy}

\section{Hyperemesis Gravidarum}

Hyperemesis gravidarum $(\mathrm{HG})$ is a severe form of nausea and vomiting seen in the first trimester. It affects $0.2-2 \%$ of all pregnancies. It can lead to dehydration; electrolyte disturbance; weight loss of greater than 5\%; thiamine deficiency; and, in severe cases if left untreated, death [8]. The exact etiology of HG remains unclear. However, it might be related to hormonal changes during pregnancy. Potential risk factors include hyperthyroidism, molar pregnancies, diabetes mellitus, history of gastrointestinal disorders, and asthma [9].

Patients develop symptoms early in the first trimester, with $60 \%$ of patients symptomatic by week 6 . Symptoms typically resolve by 20 weeks of gestation [10]. The presence of confusion and nystagmus should raise the concern of Wernicke's encephalopathy due to thiamine deficiency. Serum aminotransferases are usually mildly elevated and rarely raised > 1000 IU. Jaundice is extremely rare in HG [11]. Oher biochemical abnormalities are commonly related to dehydration and include hypokalemia, hypophosphatemia, and acute kidney injury. Biochemical abnormalities typically resolves with hydration and the resolution of vomiting. Persistent abnormalities despite the resolution of symptoms should prompt evaluation of an alternative etiology.

The treatment is largely supportive. Patients may require frequent hospitalization for IV hydration and antiemetics. Nutritional and vitamins supplementation especially thiamine is required [4]. In the absence of severe malnutrition, hyperemesis gravidarum is not associated with significant adverse pregnancy outcomes [12]. The risk of recurrence is high with subsequent pregnancies [13].

\section{Intrahepatic Cholestasis of Pregnancy}

Intrahepatic cholestasis of pregnancy (ICP) is the most commonly encountered liver disease unique to pregnancy. It is characterized by pruritus, elevated serum bile acids level, and rapid resolution of symptoms after delivery, typically in 4-6 weeks [14]. The incidence of ICP varies according to ethnicity and geographic distribution. The general reported incidence is $0.2-2 \%$. The highest incidence has been reported in South America and Europe [15]. The incidence of ICP is higher among pregnant women infected with hepatitis $\mathrm{C}$ virus which raises the concern for the need to screen all patients with ICP for HCV $[16,17]$. The other risk factors for ICP include personal or family history, multiple pregnancies, advanced maternal age, and cholelithiasis. Estrogen and progesterone, genetic mutations in bile acid transport proteins and environmental factors play a role in the pathogenesis of ICP [18].

Patients with ICP typically present with pruritus in the late second or third trimester of pregnancy. However, the development of pruritus at any stage of pregnancy especially after week 7 should raise the suspicion of ICP given reports of early presentations [15]. Pruritus of the palms and soles is the key symptom, although it can be generalized. The pruritus can be severe and may result in sleep disturbance in some cases. Skin rash is typically absent, and its presence apart from excoriations from scratching may indicate an alternative etiology of pruritus. Jaundice is rarely seen in patients with ICP. If jaundice develops, it tends to be mild and the bilirubin rarely exceeds $6 \mathrm{mg} / \mathrm{dl}$ [19].

The diagnosis of ICP is based on characteristic clinical features and elevated serum bile acids ( $>11 \mathrm{umol} / \mathrm{L}$ ). Serum bile acids levels above $40 \mu \mathrm{mol} / \mathrm{L}$ are associated with adverse fetal outcomes $\left[20 \bullet^{\circ}\right]$. Serum aminotransferases are typically elevated in the majority of cases [14]. Aminotransferase levels $>1000 \mathrm{U} / \mathrm{I}$ have been reported [6]. Liver biopsy is rarely needed to confirm diagnosis. When performed, it usually reveals cholestasis with preserved portal tracts [21].

Early recognition and management is of paramount importance given significant adverse fetal outcomes particularly if serum bile acids are significantly elevated. The treatment requires a multidisciplinary team approach with two main goals, alleviating maternal symptoms and reducing perinatal morbidity and mortality. Ursodeoxycholic acid (UDCA) is the firstline treatment. Based on a meta-analysis published in 2012, UDCA resulted in complete resolution of pruritus in $42 \%$ of patients and $61 \%$ had symptoms improvement. Furthermore, UDCA was associated with normalization or improvement in ALT and serum total bile acid levels and may improve fetal outcomes [22]. The dose of UDCA is $10-15 \mathrm{mg} / \mathrm{kg}$ of maternal body weight. It is usually well tolerated and has an excellent safety profile. For refractory pruritus, one of the following drugs can be added: cholestyramine, rifampin, and Sadenosyl-methionine [23]. Cholestyramine binds other medications which could lead to UDCA malabsorption so it should be given $2-4 \mathrm{~h}$ before or after UCDA. Dexamethasone is administered when delivery is considered to promote fetal lung maturity.

Serious adverse fetal outcomes are the main concern including fetal distress, preterm labor, prematurity, and intrauterine death. Serum bile acids levels of $100 \mu \mathrm{mol} / \mathrm{L}$ or greater are associated with increased risk of intrauterine death [18]. Maternal outcomes are excellent with resolution of pruritus a 
few days post-delivery as well as normalization of serum bile acids and liver enzymes. Most societies recommend delivery at week 37 given the increased risk of intrauterine fetal demise in the last 4 weeks of pregnancy [6]. The risk of recurrence in subsequent pregnancies is high and may reach up to $90 \%$ [24].

\section{HELLP Syndrome}

HELLP syndrome is characterized by hemolysis, elevated liver enzymes, and a low platelet count and is a severe form of pre-eclampsia. The literature has expanded since then regarding the incidence, pathophysiology, risk factors, pregnancy outcome, and treatment of this syndrome. HELLP affects approximately $0.2-0.8 \%$ of all pregnancies. The incidence of HELLP is $8-24 \%$ among patients with severe pre-eclampsia [25]. It typically presents in the third trimester; however, onethird of patients develop HELLP in the first week post-partum. The onset of symptoms in the second trimester is not uncommon.

Inadequate immune tolerance results in damage to the invading fetal trophoblast early in pregnancy is hypothesized to be the initial phenomenon of HELLP. Endothelial injury and fibrin deposition may cause the microangiopathic process of HELLP. Subsequently, liver hemorrhage and necrosis may develop [26]. Maternal risk factors include high body mass index, infertility treatment, and a previous history of preeclampsia or HELLP [26]. HELLP syndrome might be the initial presentation of antiphospholipid-antibody syndrome [27].

The clinical presentation is variable. Patients might be asymptomatic on presentation or may present with nonspecific symptoms such as malaise and nausea. Other symptoms that patients may report are abdominal pain, headache, and edema. Approximately, two-thirds of patients may have hypertension on presentation. Lack of proteinuria and normal blood pressure on presentation can be seen as well [28].

Microangiopathic hemolytic anemia is the hallmark of HELLP. The classical findings of hemolysis are abnormal peripheral blood smear, elevated unconjugated bilirubin, low haptoglobin, elevated lactate dehydrogenase, and anemia [29]. Aminotransferases levels vary from mild elevation to levels $>$ 1000 IU. Two classification systems exist for diagnosis of HELLP. Tennessee Classification requires the following for the diagnosis: AST > $70 \mathrm{IU} / \mathrm{L}, \mathrm{LDH}>600 \mathrm{IU} / \mathrm{L}$, and platelets $<100,000$ cells/L. The Tennessee Classification defines HELLP as "true" or "complete" if all three criteria are present. Partial or incomplete HELLP is used when one or more of the parameters is missing [30]. The second system is the Mississippi classification which further classifies HELLP based on the nadir platelet count as illustrated in Table 1. Liver imaging should be performed in all patients with suspected HELLP.
Table 1 Mississippi classification which further classifies HELLP based on the nadir platelet count

\begin{tabular}{ll}
\hline HELLP class & Mississippi classification \\
\hline 1 & Platelets $<50 \times 10^{9} / \mathrm{L}$ \\
& AST or ALT $\geq 70 \mathrm{IU} / \mathrm{L}$ \\
& LDH $\geq 600 \mathrm{IU} / \mathrm{L}$ \\
2 & Platelets $<100 \times 10^{9} / \mathrm{L}$ \\
& AST or ALT $\geq 70 \mathrm{IU} / \mathrm{L}$ \\
& LDH $\geq 600 \mathrm{IU} / \mathrm{L}$ \\
& Platelets $<150 \times 10^{9} / \mathrm{L}$ \\
& AST or ALT $\geq 40 \mathrm{IU} / \mathrm{L}$ \\
& LDH $\geq 600 \mathrm{IU} / \mathrm{L}$ \\
\hline
\end{tabular}

HELLP is associated with significant mortality and morbidity. Immediate recognition and management are crucial given potentially life-threatening complications as a results of end organ damage. Deaths in patients with HELLP occur mostly in class 1 . Delayed diagnosis appears to be a key factor in maternal mortality [31].

Management of HELLP requires close coordination between obstetricians and hepatologists. Patients must be admitted to a monitored unit. Supportive treatment should be initiated immediately. Blood pressure control is necessary which can be achieved via IV medications including labetalol or hydralazine. Magnesium sulfate can be given to prevent seizures. Supportive transfusion should be considered to correct coagulopathy. Delivery is the only definitive therapy. The risk of recurrence is high with subsequent pregnancies [1].

\section{Hepatic Infarction, Parenchymal Hemorrhage, and Subcapsular Hematoma}

Hepatic infarction is a rare complication of HELLP. Patients usually presents with abdominal pain, fever, and other symptoms related to HELLP. Laboratory evaluation reveals significantly elevated transaminases. Cross-sectional studies with either CT or MRI are required to make the diagnosis. Patients with antiphospholipid syndrome are the highest risk group as demonstrated in case series [32]. The presence of abdominal pain, fever, and ALT or AST that is $>1000 \mathrm{U} / \mathrm{L}$ should raise the suspicion for hepatic parenchymal hemorrhage and subcapsular hematoma. Supportive treatment is recommended for stable patients. Angiographic embolization or surgical interventions should be performed in patients with ongoing signs of bleeding or hemodynamic instability [33].

\section{Hepatic Rupture}

Hepatic rupture is a rare but potentially fatal complication of HELLP. It is usually preceded by parenchymal hemorrhage. Characteristic symptoms and signs are abdominal pain, nausea, vomiting, right shoulder pain, and hypovolemic shock. 
High index of suspicion and immediate intervention are warranted because delayed diagnosis is associated with poor outcome. Cross sectional imaging studies should be obtained as soon as possible. Patients should be transferred immediately to a specialized center with liver transplantation capabilities. Supportive treatment is appropriate as initial therapy. Embolization of hepatic artery should be performed if patients develop signs of hemodynamic instability. If embolization fails, patients may undergo operative management which may include peri-hepatic packing, drainage, and hepatic artery ligation, with or without hepatic resection. Liver transplantation for patients who fail all modalities has been reported in the literature [34].

\section{Acute Fatty Liver of Pregnancy}

Acute fatty liver of pregnancy (AFLP) is a life-threatening medical and obstetric emergency that occurs in the third trimester. It is characterized by microvesicular steatosis resulting in progressive liver failure and development of hepatic encephalopathy. AFLP carries significant maternal and fetal mortality [1]. The incidence of AFLP was variable in older studies. A study from the UK published in 2008 revealed that the incidence in the UK is 1 in 20,000 pregnancies [35].

AFLP is a mitochondrial disease. Long-chain 3hydroxyacyl-coenzyme A (CoA) dehydrogenase (LCHAD) catalyzes the step in the $\beta$-oxidation of fatty acids in the mitochondria. If the fetus is homozygous for LCHAD deficiency, the intermediate metabolites of fatty acid metabolism will enter the maternal circulation. Maternal hepatotoxicity occur when the mother is heterozygous for LCHAD deficiency [36].

Patients with AFLP present with a wide range of symptoms. Nausea and vomiting are seen in approximately $75 \%$ of patients whereas jaundice appears in 37\%. Hepatic encephalopathy and coma may be seen in severe cases. Risk factors include multigravidas, increased maternal age, low body mass index, and multiple gestations [37, 38].

Multiple laboratory abnormalities are seen in AFLP. Aminotransferases levels range from normal to $>1000$ IU and bilirubin is usually below $5 \mathrm{mg} / \mathrm{dL}$ on presentation. Other commonly seen abnormalities are DIC, anemia, acute kidney injury, high ammonia, and hypoglycemia. Liver biopsy is a useful tool to confirm the diagnosis although not routinely performed. Furthermore, patients might be critically ill with coagulopathy which preclude the biopsy. Histologically, microvesicular fatty infiltration predominantly in zone 3 with lobular disarray and mild portal inflammation with cholestasis is seen [1].

The Swansea criteria is a very helpful diagnostic tool to diagnose AFLP based on clinical criteria (Table 2) [2]. The number needed to make the diagnosis is between 6 and 9 . When Swansea criteria were applied to patients who underwent liver biopsy, the sensitivity and specificity were
$100 \%$ and $57 \%$, respectively. The positive predictive value was $85 \%$ and the negative predicative value was $100 \%$ [39].

Prompt recognition and management are essential given very high maternal and fetal mortalities. Immediate delivery is the key in managing patients with AFLP irrespective of gestational age. Correction of coagulopathy is required prior to delivery. Most patients recover within 4 weeks although the cholestatic hepatitis phase might be prolonged. Liver transplantation can be considered for patients with acute liver failure. Recurrence in subsequent pregnancies has been reported and counseling of affected women is crucial [33].

Children with LCHAD deficiency may present with nonketotic hypoglycemia during fasting and hepatic encephalopathy [36]. A multidisciplinary team approach and effective communication are of paramount importance.

The differential diagnosis of diseases unique to pregnancy based on trimester is illustrated in Fig. 1.

\section{Coincident Liver Diseases with Pregnancy}

\section{Acute Viral Hepatitis}

An acute rise in aminotransferases during pregnancy should raise the suspicion for acute viral hepatitis. The incidence of acute hepatitis A virus (HAV) in pregnancy is rare. Maternal and fetal outcomes are excellent. However, HAV can be associated with preterm labor and gestational complications if it occurs in the third trimester [40]. Management of acute HAV is supportive. The hepatitis A vaccine is considered safe during pregnancy and should be considered for high risk groups [41]. Breastfeeding is not discouraged even during acute infection [42].

Hepatitis E virus (HEV) is a common cause of acute viral hepatitis particularly in endemic areas of Asia, Africa, and Central America. It is usually a self-limited disease and spontaneous clearance is common. However, the HEV course in pregnant women is different and potentially fatal. The disease course is severe during pregnancy with a mortality rate of 10 $25 \%$. Maternal mortality occurs in the third trimester [43]. Maternal complications include progression to fulminant liver failure, pre-eclampsia, and antepartum hemorrhage. Intrauterine fetal demise is common [44]. The treatment is usually supportive. Ribavirin is the drug of choice when antiviral therapy is indicated; however, it is contraindicated in pregnancy due to teratogenic effects. An effective vaccine has developed, but is not widely available and the safety in pregnancy is yet to be determined [45].

Herpes simplex virus (HSV) rarely causes acute hepatitis in pregnancy. Prompt diagnosis is critical because it carries a significant risk in pregnant women. Symptoms of HSV are nonspecific. Patients may present with fever, nausea, vomiting, and abdominal pain. Mucocutaneous lesions are 
Table 2 Swansea criteria for diagnosis of acute fatty liver of pregnancy

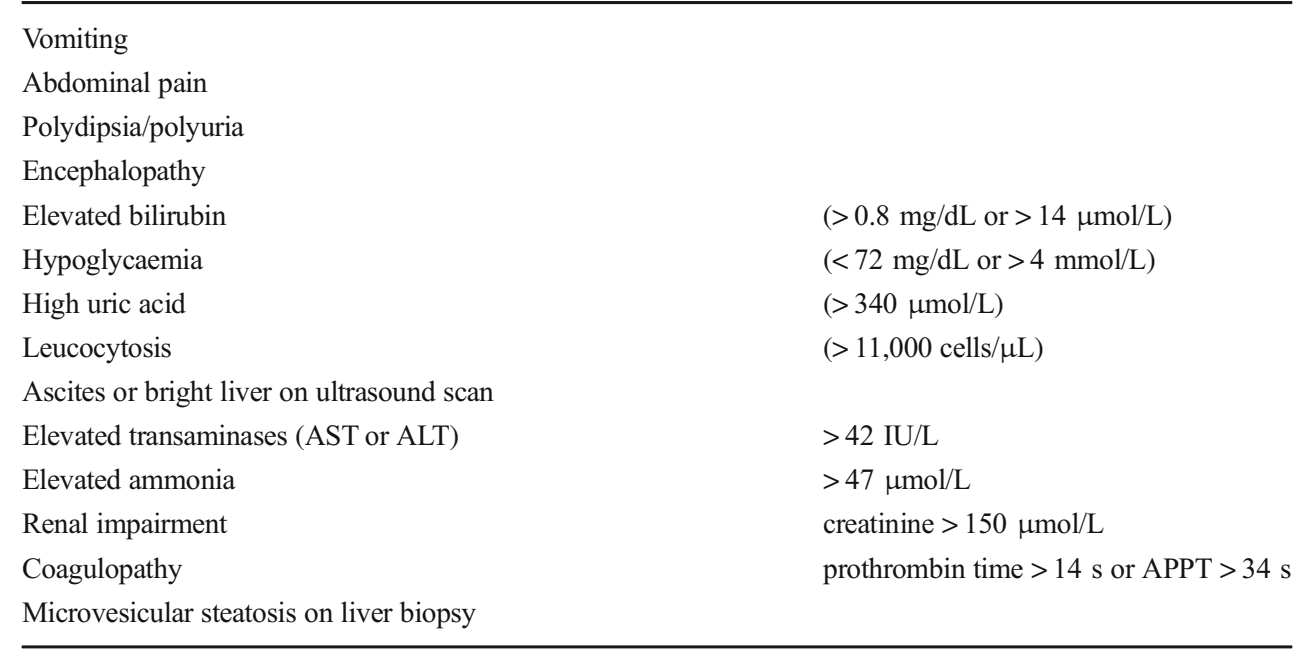

seen in less than $50 \%$ of patients [6]. The mortality rate of HSV hepatitis in pregnancy is approximately 40\% [46]. Patients present with acute severe (anicteric) hepatitis or acute liver failure in the third trimester. Significantly elevated aminotransferases, thrombocytopenia, and normal bilirubin are commonly seen. The absence of cutaneous lesions does not exclude diagnosis. Treatment with acyclovir should be initiated immediately before confirmatory tests are obtained.

\section{Budd-Chiari Syndrome}

Budd-Chiari syndrome (BCS) is a vascular disease of the liver characterized by hepatic venous outflow obstruction. The disease may progress to cirrhosis if left untreated and the hepatic sinusoidal pressure is not reduced. Risk factors for development of BCS include trauma, hypercoagulable conditions, myeloproliferative disorders, oral contraceptive pills, and pregnancy. BCS usually presents with abdominal pain, hepatomegaly, and ascites. A Doppler ultrasound of the liver is sufficient in most cases to establish the diagnosis [47].

Successful pregnancies in the setting of known BCS have been reported in the literature. Multidisciplinary care is required for patients with BCS before, during, and after pregnancy. Hypercoagulable conditions should be treated adequately because the pregnancy may exacerbate these conditions [48].

\section{Pre-existing Liver Diseases and Pregnancy}

\section{Cirrhosis and Portal Hypertension}

Pregnancy in the setting of cirrhosis is an uncommon event although the worldwide incidence is unknown. Several metabolic and endocrine factors play a role in reduced fertility in patients with cirrhosis. The development of liver

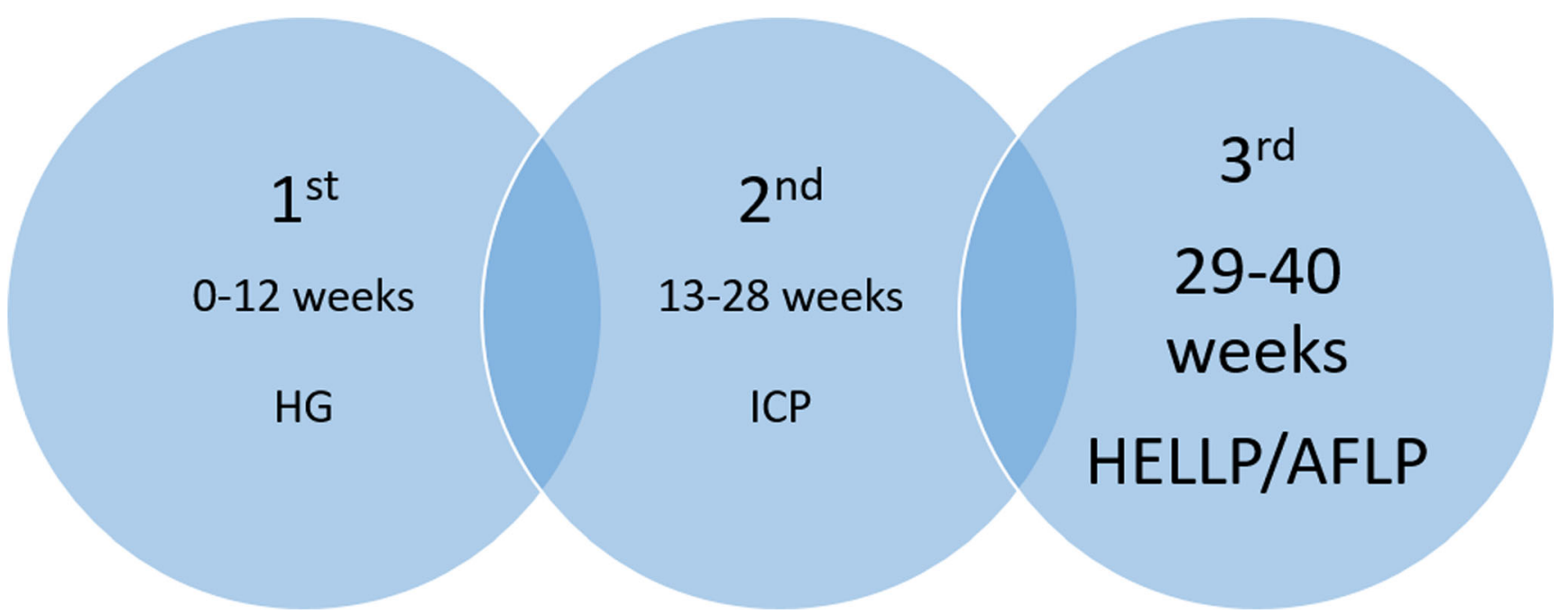

Fig. 1 HG, hyperemesis Gravidarum; ICP, intrahepatic cholestasis of pregnancy; AFLP, acute fatty liver of pregnancy 
decompensation, hepatic encephalopathy, and worsening portal hypertension represents the main challenges facing pregnant women with cirrhosis [49]. The risk of cesarean section is high based on a recent population based study [50]. The main fetal risks are spontaneous abortion, premature birth, and still births [51].

Variceal bleeding is a catastrophic complication during pregnancy. It has been reported in 18-32\% of pregnant women with cirrhosis [49]. The risk of variceal bleeding is high during the second trimester and delivery [1]. The mortality rate may reach $50 \%$ based on historic studies [52]. The management of bleeding esophageal varices is similar to nonpregnant patients. Pregnant women may undergo endoscopic band ligation, or TIPS when necessary. Because of the vasoconstriction property, octreotide infusion carries a theoretical risk of uterine ischemia and placental abruption so it must be used with caution.

Unplanned pregnancies can occur in patients with cirrhosis and may lead to serious complications. Therefore, we encourage women at childbearing age to discuss contraceptive methods with their hepatologists and gynecologists. There are no restrictions on the use of oral hormonal contraceptive pills in patients with compensated cirrhosis. However, their use is discouraged in patients with severely decompensated liver disease [53].

Patients with compensated cirrhosis who desire to get pregnant should be medically optimized before pregnancy. Cirrhosis must be compensated and the portal hypertension is adequately controlled. Patients must undergo EGD for esophageal varices screening. If no varices are identified, the EGD should be repeated in the second trimester. Esophageal band ligation is the treatment of choice for medium and large varices found during screening endoscopy either prepregnancy or in the second trimester. When indicated, propranolol is the preferred non selective beta blocker agent during pregnancy. The data is lacking on the role of prophylactic TIPS [6].

\section{Splenic Artery Aneurysm Rupture}

Splenic artery aneurysm (SAA) is commonly encountered in young women, when compared to other visceral arterial aneurysms. Contributing factors include pregnancy and portal hypertension. The vast majority of patients are asymptomatic. Symptomatic patients usually present with abdominal pain, syncope, or shock. Ruptured SAA is a catastrophic complication and occurs in $20-50 \%$ of cases during pregnancy with reported maternal mortality reaching $75 \%$. The risk of rupture increases when the aneurysm is larger than $2.5 \mathrm{~cm}$ [54]. The diagnosis is usually confirmed by crosssectional imaging studies or duplex ultrasound. However, unstable patients may undergo emergency surgery before radiological investigations.
Resuscitation and supportive transfusion are the initial key elements in the management of ruptured SAA. Emergency laparotomy with splenectomy and ligation of the splenic artery is usually performed. Angioembolization is a less-invasive option that can be pursued in highly selected patients [55].

\section{Hepatic Adenomas}

Women with known hepatic adenomas are at high risk for rapid growth during pregnancy secondary to the associated high estrogen state. Hepatic adenomas should be monitored routinely during pregnancy given this risk of rapid growth and rupture. Surgical resection or angioembolization should be considered for large adenomas $(>5 \mathrm{~cm})$ prior to conception [6]. If treatment is required during pregnancy, interventions should be carried out during the second trimester.

Based on the limited available evidence, pregnancy is not discouraged in patients with adenomas less than $5 \mathrm{~cm}$ if there is no evidence of complications. Serial follow with imaging studies is recommended to ensure stability [56].

\section{Chronic Hepatitis B Virus Infection}

Multiple societies recommend screening all pregnant women for HBV infection early in pregnancy [57]. This is a key element to reducing mother-to-child transmission as part of the global effort to eliminate chronic hepatitis B. HBV vaccine is effective and safe during pregnancy. Per most recent AASLD guidelines, HBV vaccine should be administered to pregnant women not immune or infected with HBV [58].

Vertical transmission continues to be a common method of HBV infection, particularly in endemic areas. It is estimated that vertical transmission represents approximately $50 \%$ of HBV infection worldwide. High maternal HBV DNA and positive $\mathrm{HBeAg}$ are the most important risk factors for mother-to-child transmission. The transmission may occur intrauterine (trans placental), following diagnostic procedures, during delivery, or during breastfeeding. The transmission is not affected by the mode of delivery. The risk for development of chronic HBV infection is $90 \%$ when acquired in infancy in contrast to $5 \%$ during adulthood $[33,57]$.

The risk of HBV transmission can be decreased by initiation of antiviral therapy in the third trimester. AASLD guidelines recommend consideration of treatment if HBV DNA is greater than $200,000 \mathrm{IU} / \mathrm{mL}$ in the second trimester in women without standard indications for HBV treatment [58]. However, a double-blinded randomized study published in 2018 showed no additional benefit of tenofovir in the third trimester if infants received HBIG and vaccine at birth [59••]. All infants born to HBV-infected mothers should receive 
hepatitis B immune globulin (HBIG) and the first dose of HBV vaccine immediately after delivery.

When antiviral therapy is indicated, tenofovir is the first drug of choice. Lamivudine is a potential alternative if tenofovir is not affordable; however, viral resistance remains a concern. Physicians should determine the mode of delivery based on obstetric indications and should not pursue cesarean delivery simply for prevention of HBV transmission. Breastfeeding is not contraindicated if immunoprophylaxis is given at birth [57].

\section{Hepatitis C Infection}

Hepatitis $\mathrm{C}$ infection is common among pregnant women with a worldwide prevalence of $8 \%$ [60]. AASLD guidelines recommend screening all pregnant women for HCV [61]. The rate of vertical transmission of $\mathrm{HCV}$ is 3-5\% [62]. HIV coinfection and high $\mathrm{HCV}$ viral load are the main risk factors for vertical transmission.

The best strategy to reduce HCV vertical transmission is to eradicate the virus before pregnancy. Per current guidelines, $\mathrm{HCV}$ treatment during pregnancy is not recommended owing to lack of safety of the currently available antiviral agents during pregnancy. Breastfeeding is not contraindicated; however, it should be avoided if the mother has cracked or bleeding nipples [61]. The mode of delivery should be based on obstetric indications.

\section{Autoimmune Hepatitis (AIH)}

Successful pregnancy outcomes have been reported in patients with AIH. However, disease flare is the major concern as it can be seen in up to $20 \%$ of patients during pregnancy and 30 $50 \%$ in the post-partum period. Therefore, preconception planning is the key. Patients must be in remission prior to pregnancy and preferably to have a stable disease for at least 1 year.

The treatment should be continued throughout pregnancy. Prednisone, budesonide, and azathioprine are the most commonly used agents to treat AIH. Prospective studies demonstrated the safety use of prednisone in pregnancy. However, the data is limited regarding budesonide. Animal studies showed a teratogenic effect of azathioprine. This effect has not been described in small studies in human beings. Therefore, continuation of azathioprine during pregnancy is recommended to maintain remission. The use of mycophenolate mofetil is contraindicated in pregnancy due to the risk of congenital malformations.

Close liver function test monitoring in the post-partum period is recommended given increased risk of flare. LFT should be monitored every 4-6 weeks for the first 3 months. Breastfeeding is generally safe for patients taking azathioprine.

\section{Liver Transplantation and Pregnancy}

Fertility is usually restored within 1 year following liver transplantation (LT). Approximately, $8 \%$ of women undergoing liver transplantation are of reproductive age. Pregnancy and delivery are feasible after LT with favorable outcomes [63]. Careful planning and close coordination between the liver transplant team and maternal-fetal medicine are key elements prior to pregnancy after LT.

Pregnancy is not recommended within the first year after LT due to the risk of acute cellular rejection (ACR). It should be planned based on excellent graft function, stable immunosuppression, and adequate control of the associated medical conditions. A recent study demonstrated that a preconception renal function predicts pregnancy outcome [64]. Pregnancy outcome is good. However, there is an increased risk of preterm delivery, low birth weight, hypertension, and pre-eclampsia [65••].

Tacrolimus monotherapy, if feasible, is the ideal immunosuppression. Cyclosporine, azathioprine, and prednisone are alternative options. Mycophenolate should be avoided due to the associated risk of congenital malformations. Graft function and calcineurin inhibitor levels should be monitored every 4 weeks until 32 weeks, then every 2 weeks until week 36 , and then weekly until delivery. ACR during pregnancy should be managed similarly to nonpregnant women. Liver biopsy to diagnose ACR can be performed during pregnancy. The evidence to support breastfeeding post-LT remains limited [66].

\section{Compliance with Ethical Standards}

Conflict of Interest The authors declare that they have no conflict of interest.

Human and Animal Rights and Informed Consent This article does not contain any studies with human or animal subjects performed by any of the authors.

Open Access This article is distributed under the terms of the Creative Commons Attribution 4.0 International License (http:// creativecommons.org/licenses/by/4.0/), which permits unrestricted use, distribution, and reproduction in any medium, provided you give appropriate credit to the original author(s) and the source, provide a link to the Creative Commons license, and indicate if changes were made.

\section{References}

Papers of particular interest, published recently, have been highlighted as:

•• Of major importance

1. Hay J. Liver disease in pregnancy. Hepatology. 2008;47(3):1067-76.

2. Ch'Ng C, Morgan M, Hainsworth I, Kingham JJ. Prospective study of liver dysfunction in pregnancy in Southwest Wales. Gut. 2002;51(6):876-80. 
3. Allen AM, Kim WR, Larson JJ, Rosedahl JK, Yawn BP, McKeon $\mathrm{K}$, et al. The epidemiology of liver diseases unique to pregnancy in a US community: a population-based study. Clin Gastroenterol Hepatol. 2016;14(2):287-94. e2.

4. Westbrook RH, Dusheiko G, Williamson CJ. J Hepatol Pregnancy Liver Dis. 2016;64(4):933-45.

5. Heller MT, Tublin ME, Hosseinzadeh K, Fargiano AJ. Imaging of hepatobiliary disorders complicating pregnancy. Am J Roentgenol. 2011;197(3):W528-W36.

6. Tran TT, Ahn J, Reau NS. ACG clinical guideline: liver disease and pregnancy. Am J Gastroenterol. 2016;111(2):176-94.

7. Ludvigsson JF, Marschall HU, Hagström H, Höijer J, Stephansson O. Pregnancy outcome in women undergoing liver biopsy during pregnancy: a nationwide population-based cohort study. Hepatology. 2018;68(2):625-33.

8. Bailit J. Gynecology. Hyperemesis gravidarium: epidemiologic findings from a large cohort. Am J Obstet Gynecol. 2005;193(3): 811-4.

9. Fell DB, Dodds L, Joseph KS, Allen VM, Butler B. Risk factors for hyperemesis gravidarum requiring hospital admission during pregnancy. Obstet Gynecol. 2006;107(2):277-84.

10. Goodwin T. Hyperemesis gravidarum. Obstet Gynecol Clin N Am. 2008;35(3):401-17.

11. Conchillo J, Pijnenborg J, Peeters P, Stockbrugger R, Fevery J, Koek G. Liver enzyme elevation induced by hyperemesis gravidarum: aetiology, diagnosis and treatment. Neth J Med. 2002;60(9):374-8.

12. Dodds L, Fell DB, Joseph KS, Allen VM, Butler B. Outcomes of pregnancies complicated by hyperemesis gravidarum. Obstet Gynecol. 2006;107(2):285-92.

13. Trogstad LI, Stoltenberg C, Magnus P, Skjærven R, Irgens L. Recurrence risk in hyperemesis gravidarum. BJOG Int J Obstet Gynaecol. 2005;112(12):1641-5.

14. European Association For The Study Of The Liver. EASL Clinical Practice Guidelines: management of cholestatic liver diseases. J Hepatol. 2009;51(2):237-67.

15. Williamson C, Geenes V. Intrahepatic cholestasis of pregnancy. Obstet Gynecol. 2014;124(1):120-33.

16. Belay $\mathrm{T}$, Woldegiorgis $\mathrm{H}$, Gress $\mathrm{T}$, Rayyan Y. Intrahepatic cholestasis of pregnancy with concomitant hepatitis $\mathrm{C}$ virus infection, Joan C Edwards S. Eur J Gastroenterol Hepatol. 2015;27(4):372-4.

17. Wijarnpreecha K, Thongprayoon C, Sanguankeo A, Upala S, Ungprasert P, Cheungpasitporn W, et al. Hepatitis C infection and intrahepatic cholestasis of pregnancy: a systematic review and meta-analysis. Clin Res Hepatol Gastroenterol. 2017;41(1):39-45.

18. Wood AM, Livingston EG, Hughes BL, Kuller JA. Intrahepatic cholestasis of pregnancy: a review of diagnosis and management. Obstet Gynecol Surv. 2018;73(2):103-9.

19. Geenes V, Williamson C. Intrahepatic cholestasis of pregnancy. World J Gastroenterol. 2009;15(17):2049-66.

20.• Garcia-Flores J, Cañamares M, Cruceyra M, Garicano A, Espada $\mathrm{M}$, Lopez A, et al. Clinical value of maternal bile acid quantification in intrahepatic cholestasis of pregnancy as an adverse perinatal outcome predictor. Gynecol Obstet Investig. 2015;79(4):222-8 This study illustrates the importance of bile acids level and identification of high-risk groups.

21. Bacq Y, Sentilhes L. Intrahepatic cholestasis of pregnancy: diagnosis and management. Clin Liver Dis. 2014;4(3):58-61.

22. Bacq Y, Sentilhes L, Reyes HB, Glantz A, Kondrackiene J, Binder $\mathrm{T}$, et al. Efficacy of ursodeoxycholic acid in treating intrahepatic cholestasis of pregnancy: a meta-analysis. Gastroenterology. 2012;143(6):1492-501.

23. Geenes V, Chambers J, Khurana R, Shemer EW, Sia W, Mandair D, et al. Rifampicin in the treatment of severe intrahepatic cholestasis of pregnancy. Eur J Obstet Gynecol Reprod Biol. 2015;189:59-63.
24. Gonzalez MC, Reyes H, Arrese M, Figueroa D, Lorca B, Andresen $\mathrm{M}$, et al. Intrahepatic cholestasis of pregnancy in twin pregnancies. J Hepatol. 1989;9(1):84-90.

25. Fitzpatrick KE, Hinshaw K, Kurinczuk JJ, Knight M. Risk factors, management, and outcomes of hemolysis, elevated liver enzymes, and low platelets syndrome and elevated liver enzymes, low platelets syndrome. Obstet Gynecol. 2014;123(3):618-27.

26. Abildgaard U, Heimdal K. Pathogenesis of the syndrome of hemolysis, elevated liver enzymes, and low platelet count (HELLP): a review. Eur J Obstet Gynecol Reprod Biol. 2013;166(2):117-23.

27. Koenig M, Roy M, Baccot S, Cuilleron M, de Filippis J-P, Cathébras P. Thrombotic microangiopathy with liver, gut, and bone infarction (catastrophic antiphospholipid syndrome) associated with HELLP syndrome. Clin Rheumatol. 2005;24(2):166-8.

28. Baxter JK, Weinstein L. HELLP syndrome: the state of the art. Obstet Gynecol Surv. 2004;59(12):838-45.

29. Sibai B. Diagnosis, controversies, and management of the syndrome of hemolysis, elevated liver enzymes, and low platelet count. Obstet Gynecol. 2004;103(5):981-91.

30. Martin JN Jr, Rose CH, Briery C. Understanding and managing HELLP syndrome: the integral role of aggressive glucocorticoids for mother and child. Am J Obstet Gynecol. 2006;195(4):914-34.

31. Isler CM, Rinehart BK, Terrone DA, Martin RW, Magann EF, Martin J Jr, et al. Maternal mortality associated with HELLP (hemolysis, elevated liver enzymes, and low platelets) syndrome. Am J Obstet Gynecol. 1999;181(4):924-8.

32. Pauzner R, Dulitzky M, Carp H, Mayan H, Kenett R, Farfel Z, et al. Hepatic infarctions during pregnancy are associated with the antiphospholipid syndrome and in addition with complete or incomplete HELLP syndrome. J Thromb Haemost. 2003;1(8): 1758-63.

33. Joshi D, James A, Quaglia A, Westbrook RH, Heneghan M. Liver disease in pregnancy. Lancet. 2010;375(9714):594-605.

34. Wicke C, Pereira PL, Neeser E, Flesch I, Rodegerdts EA, Becker HD. Subcapsular liver hematoma in HELLP syndrome: evaluation of diagnostic and therapeutic options - a unicenter study. Am J Obstet Gynecol. 2004;190(1):106-12.

35. Knight M, Nelson-Piercy C, Kurinczuk JJ, Spark P, Brocklehurst P. A prospective national study of acute fatty liver of pregnancy in the UK. Gut. 2008;57(7):951-6.

36. Ibdah JA, Bennett MJ, Rinaldo P, Zhao Y, Gibson B, Sims HF, et al. A fetal fatty-acid oxidation disorder as a cause of liver disease in pregnant women. N Engl J Med. 1999;340(22):1723-31.

37. Liu J, Ghaziani TT, Wolf JL. Acute fatty liver disease of pregnancy: updates in pathogenesis, diagnosis, and management. Am J Gastroenterol. 2017;112(6):838-46.

38. Fesenmeier MF, Coppage KH, Lambers DS, Barton JR, Sibai BM. Acute fatty liver of pregnancy in 3 tertiary care centers. Am J Obstet Gynecol. 2005;192(5):1416-9.

39. Goel A, Ramakrishna B, Zachariah U, Ramachandran J, Eapen C, Kurian G, et al. How accurate are the Swansea criteria to diagnose acute fatty liver of pregnancy in predicting hepatic microvesicular steatosis? Gut. 2011;60(1):138-9.

40. Elinav E, Ben-Dov IZ, Shapira Y, Daudi N, Adler R, Shouval D, et al. Acute hepatitis A infection in pregnancy is associated with high rates of gestational complications and preterm labor. Gastroenterology. 2006;130(4):1129-34.

41. Duff B, Duff P. Hepatitis A vaccine: ready for prime time. Obstet Gynecol. 1998;91(3):468-71.

42. Rac MW, Sheffield JS. Prevention and management of viral hepatitis in pregnancy. Obstet Gynecol Clin. 2014;41(4):573-92.

43. Kamar N, Bendall R, Legrand-Abravanel F, Xia N-S, Ijaz S, Izopet J, et al. Hepatitis e. Lancet. 2012;379(9835):2477-88. 
44. Patra S, Kumar A, Trivedi SS, Puri M, Sarin S. Maternal and fetal outcomes in pregnant women with acute hepatitis $\mathrm{E}$ virus infection. Ann Intern Med. 2007;147(1):28-33.

45. Zhang J, Zhang XF, Zhou C, Wang ZZ, Huang SJ, Yao X, et al. Protection against hepatitis $\mathrm{E}$ virus infection by naturally acquired and vaccine-induced immunity. Clin Microbiol Infect. 2014;20(6): O397-405.

46. Kourtis AP, Read JS, Jamieson DJ. Pregnancy and infection. N Engl J Med. 2014;370(23):2211-8.

47. Menon KN, Shah V, Kamath P. The Budd-Chiari syndrome. N Engl J Med. 2004;350(6):578-85.

48. Rautou P-E, Angermayr B, Garcia-Pagan J-C, Moucari R, PeckRadosavljevic M, Raffa S, et al. Pregnancy in women with known and treated Budd-Chiari syndrome: maternal and fetal outcomes. J Hepatol. 2009;51(1):47-54.

49. Tan J, Surti B, Saab S. Pregnancy and cirrhosis. Liver Transpl. 2008;14(8):1081-91.

50. Hagström H, Höijer J, Marschall HU, Williamson C, Heneghan MA, Westbrook RH, et al. Outcomes of pregnancy in mothers with cirrhosis: a national population-based cohort study of 1.3 million pregnancies. Hepatol Commun. 2018;2(11):1299-305.

51. Aggarwal N, Negi N, Aggarwal A, Bodh V, Dhiman R. Pregnancy with portal hypertension. J Clin Exp Hepatol. 2014;4(2):163-71.

52. Cheng Y-S. Pregnancy in liver cirrhosis and/or portal hypertension. Am J Obstet Gynecol. 1977;128(7):812-22.

53. Kapp N. WHO provider brief on hormonal contraception and liver disease. Contraception. 2009;80(4):325-6.

54. Ha JF, Phillips M, Faulkner K. Splenic artery aneurysm rupture in pregnancy. Eur J Obstet Gynecol Reprod Biol. 2009;146(2):133-7.

55. Sadat U, Dar O, Walsh S, Varty K. Splenic artery aneurysms in pregnancy-a systematic review. Int J Surg. 2008;6(3):261-5.

56. Marrero JA, Ahn J, Rajender Reddy K, Americal College of G. ACG clinical guideline: the diagnosis and management of focal liver lesions. Am J Gastroenterol. 2014;109(9):1328-47 quiz 48.

57. Dionne-Odom J, Tita AT, Silverman NS, Society for Maternal-Fetal Medicine (SMFM). \# 38: hepatitis B in pregnancy screening, treatment, and prevention of vertical transmission. Am J Obstet Gynecol. 2016;214(1):6-14.
58. Terrault NA, Lok AS, McMahon BJ, Chang KM, Hwang JP, Jonas $\mathrm{MM}$, et al. Update on prevention, diagnosis, and treatment of chronic hepatitis B: AASLD 2018 hepatitis B guidance. Hepatology. 2018;67(4):1560-99.

59.• Jourdain G, Ngo-Giang-Huong N, Harrison L, Decker L, Khamduang W, Tierney C, et al. Tenofovir versus placebo to prevent perinatal transmission of hepatitis B. N Engl J Med. 2018;378(10):911-23 This study shows no additional benefits of HBV in this group of patients which is against the current AASLD guidlines.

60. Hughes BL, Page CM, Kuller JA. Hepatitis C in pregnancy: screening, treatment, and management. Am J Obstet Gynecol. 2017;217(5):B2-B12.

61. AASLD-IDSA. Recommendations for testing, managing, and treating hepatitis C. http://www.hovguidelines.org. Accessed 5/25/ 2019.

62. Mast EE, Hwang L-Y, Seto DS, Nolte FS, Nainan OV, Wurtzel H, et al. Risk factors for perinatal transmission of hepatitis $\mathrm{C}$ virus (HCV) and the natural history of HCV infection acquired in infancy. J Infect Dis. 2005;192(11):1880-9.

63. Ramirez CB, Doria CJ. Pregnancy after liver transplantation. Best Pract Res Clin Obstet Gynaecol. 2014;28(8):1137-45.

64. Lim TY, Gonsalkorala E, Cannon MD, Gabeta S, Penna L, Heaton ND, et al. Successful pregnancy outcomes following liver transplantation is predicted by renal function. Liver Transpl. 2018;24(5):606-15.

65.• Deshpande NA, James NT, Kucirka LM, Boyarsky BJ, GaronzikWang JM, Cameron AM, et al. Pregnancy outcomes of liver transplant recipients: a systematic review and meta-analysis. Liver Transplantation. 2012;18(6):621-9 This study will help physicians in idnetifying high-risk groups after liver transplantation.

66. Lucey MR, Terrault N, Ojo L, Hay JE, Neuberger J, Blumberg E, et al. Long-term management of the successful adult liver transplant: 2012 practice guideline by AASLD and the American Society of Transplantation. Hepatology. 2012;19(1):3-26.

Publisher's Note Springer Nature remains neutral with regard to jurisdictional claims in published maps and institutional affiliations. 